Pacific Journal of Mathematics

THE MAXIMAL RIGHT QUOTIENT SEMIGROUP OF A
STRONG SEMILATTICE OF SEMIGROUPS 


\title{
THE MAXIMAL RIGHT QUOTIENT SEMIGROUP OF A STRONG SEMILATTICE OF SEMIGROUPS
}

\author{
ANTONIO M. LOPEZ, JR.
}

Let $S$ be a strong semilattice $Y$ of monoids. If $S$ is right nonsingular then $Y$ is nonsingular. The converse is true when $S$ is a sturdy semilattice $Y$ of right cancellative monoids. Should $S$ have trivial multiplication then each monoid of more than one element has as its index an atom of $Y$. Finally, if $S$ is a right nonsingular strong semilattice $Y$ of principal right ideal Ore monoids with onto linking homomorphisms then $Q(S)$, the maximal right quotient semigroup of $S$, is a semilattice $Q(Y)$ of groups.

1. Introduction. Let $Y$ be a semilattice and let $\left\{S_{\alpha}\right\}_{\alpha \in Y}$ be a collection of pairwise disjoint semigroups. For each pair $\alpha, \beta \in Y$ with $\alpha \geqq \beta$, let $\psi_{\alpha, \beta}: S_{\alpha} \rightarrow S_{\beta}$ be a semigroup homomorphism such that $\psi_{\alpha, \alpha}$ is the identity mapping and if $\alpha>\beta>\gamma$ then $\psi_{\alpha, \gamma}=$ $\psi_{\beta, \gamma} \psi_{\alpha, \beta}$. Let $S=\mathbf{U}_{\alpha \in Y} S_{\alpha}$ with multiplication

$$
a * b=\psi_{\alpha, \alpha \beta}(a) \psi_{\beta, \alpha \beta}(b)
$$

for $a \in S_{\alpha}$ and $b \in S_{\beta}$. The semigroup $S$ is called a strong semilattice $Y$ of semigroups $S_{\alpha}$. If, in addition, each $\psi_{\alpha, \beta}$ is one-to-one then $S$ is called a sturdy semilattice of semigroups. The basic terminology in use throughout this paper can be found in [1], [7], and [9]. Note that a semilattice of groups [1, p. 128] is a strong semilattice of semigroups. In [6], McMorris showed that if $M$ is a semilattice $X$ of groups $G_{i}$, then $Q(M)$, the maximal right quotient semigroup of $M$, is also a semilattice of groups. Hinkle [2] constructed $Q(M)$ and showed that its indexing semilattice is $Q(X)$.

Let $S$ be a semigroup with 0 . A right ideal $D$ of $S$ is dense if for each $s_{1}, s_{2}, s \in S$ with $s_{1} \neq s_{2}$, there exists an element $d \in D$ such that $s_{1} d \neq s_{2} d$ and $s d \in D$. A right ideal $L$ of $S$ is $\cap$-large if for each nonzero right ideal $R$ of $S, R \cap L \neq\{0\}$. It is easy to see that dense implies $\cap$-large. If each $\cap$-large right ideal of $S$ is also dense then $S$ is said to be right nonsingular. If a semigroup is commutative or each one-sided ideal is two-sided then we will use the term nonsingular. Let $T$ be a right $S$-system with 0[5] then the singular congruence $\psi_{T}$ on $T$ is a right congruence defined for $a, b \in T$ by $a \psi_{T} b$ if and only if $a s=b s$ for all $s$ in an $\cap$-large right ideal of $S$. McMorris [8] showed that $\psi_{S}=i_{S}$, the identity congruence on $S$, if and only if $S$ is right nonsingular.

Recently it has been shown [4], [5] that if $S$ is a commutative 
nonsingular semigroup then $Q(S)$ is a semilattice of groups. However, since $S$ is commutative it is uniquely expressible as a semilattice $Y$ of archimedian semigroups [1, p. 135]. Thus we investigate right nonsingular strong semilattices of semigroups.

Henceforth we require that both $S$ and $Y$ be semigroups with 0 . If for $\alpha \in Y, S_{\alpha}$ is a monoid then the identity will be denoted by $e_{\alpha}$. Also a semigroup homomorphism which takes the identity of one semigroup to the identity of the other is called a monoid homomorphism.

LEMMA 1.1. If $S$ is a strong semilattice $Y$ of right cancellative monoids $S_{\alpha}$, then for each $\alpha, \beta \in Y$ with $\alpha \geqq \beta, \psi_{\alpha, \beta}$ is a monoid homomorphism and $Y$ is isomorphic to the semilattice $E$ of idempotents of $S$.

LEMMA 1.2. Let $S$ be a strong semilattice $Y$ of monoids $S_{\alpha}$ with $\psi_{\alpha, \beta}$ a monoid homomorphism for $\alpha \geqq \beta \in Y$. If $L$ is an $\cap-$ large right ideal of $S$, then $A=\left\{\sigma \in Y \mid L \cap S_{\sigma} \neq \varnothing\right\}$ is an $\cap$-large ideal of $Y$.

Proof. To see that $A$ is $\cap$-large let $R$ be a nonzero ideal of $Y$ and define $B=\bigcup_{\tau \in R} S_{\tau}$. Let $t \in B \cap S_{\beta}$ and $s \in S_{\sigma}$ for some $\beta \in R$ and $\sigma \in Y$. Then $t * s=\psi_{\beta, \sigma \beta}(t) \psi_{\sigma, \sigma \beta}(s) \in S_{\sigma \beta}$. But $S_{\sigma \beta} \subseteq B$ since $\beta \in R$ an ideal of $Y$. Dually we can show that $s * t \in S_{o \beta}$ and so $B$ is a twosided ideal of $S$. Since $L$ is an $\cap$-large right ideal of $S$ then $L \cap B \neq\{0\}$ so there exists $0 \neq r \in L \cap B$. But then $r \in S_{\hat{o}}$ for $0 \neq \delta \in R$ and so $0 \neq \delta \in A \cap R$ and $A$ is $\cap$-large. It is easy to show that $A$ is an ideal of $Y$.

LEMMA 1.3. Let $S$ be a strong semilattice $Y$ of monoids $S_{\alpha}$ with $\psi_{\alpha, \beta}$ a monoid homomorphism for $\alpha \geqq \beta \in Y$. If $T$ is an $\cap-$ large ideal of $Y$, then $L=\bigcup_{\beta \in T} S_{\beta}$ is an $\cap$-large ideal of $S$.

Proof. We saw in the proof of Lemma 1.2 that $L$ is an ideal of $S$. To see that $L$ is $\cap$-large we let $B$ be a nonzero right ideal of $S$, and define $R=\left\{\sigma \in Y \mid B \cap S_{\sigma} \neq \varnothing\right\}$. Since $R$ is a nonzero ideal of $Y$ and $T$ is $\cap$-large then $R \cap T \neq\{0\}$. Thus there exists $0 \neq \delta \in R \cap T$ for which $S_{\delta} \subseteq L$, and so there exists $0 \neq t \in B \cap L$.

2. Right nonsingular strong semilattices of semigroups. In studying a semigroup $M$ which is a semilattice $X$ of groups $G_{\hat{o}}$, Johnson and McMorris [3] showed that if $M$ is nonsingular then the set $E$ of idempotents of $M$ is a nonsingular semilattice. Note that under these conditions the idempotents of $M$ are central, every 
one-sided ideal is two-sided, and $X$ is isomorphic to $E$. Here we consider a weaker structure and obtain the results of Johnson and McMorris.

THEOREM 2.1. Let $S$ be a strong semilattice $Y$ of monoids $S_{\alpha}$ with $\psi_{\alpha, \beta}$ a monoid homomorphism for $\alpha \geqq \beta \in Y$. If $S$ is right nonsingular, then $Y$ is nonsingular.

Proof. Let $T$ be an $\cap$-large ideal of $Y$ and define $L=\bigcup_{\beta \in T} S_{\beta}$. Since $S$ is right nonsingular then $L$ is a dense right ideal of $S$ for, by Lemma 1.3, $L$ is an $\cap$-large right ideal of $S$. Let $\alpha, \beta \in Y$ such that $\alpha \neq \beta$. Then $e_{\alpha} \neq e_{\beta}$ and there exists an $x \in L$ such that $e_{\alpha} * x \neq$ $e_{\beta} * x$ where $x \in S_{\delta}$. Thus $\delta \in T$ and $\alpha \delta \neq \beta \delta$ for if otherwise

$$
\begin{aligned}
& e_{\alpha} * x=\psi_{\alpha, \alpha \delta}\left(e_{\alpha}\right) \psi_{\delta, \alpha \delta}(x)=\psi_{\hat{\delta}, \alpha \delta}(x) \\
& \psi_{\hat{\delta}, \beta \delta}(x)=\psi_{\beta, \beta \delta}\left(e_{\beta}\right) \psi_{\delta, \beta \delta}(x)=e_{\beta} * x
\end{aligned}
$$

which is a contradiction. Thus $T$ is dense in $Y$.

THEOREM 2.2. Let $S$ be a sturdy semilattice $Y$ of right cancellative monoids $S_{\alpha}$. If $Y$ is nonsingular, then $S$ is right nonsingular.

Proof. Let $L$ be an $\cap$-large right ideal of $S$ and let $x \neq y$, $z \in S$. Since $L$ is $\cap$-large then $z^{-1} L=\{s \in S \mid z * s \in L\}$ is an $\cap$-large right ideal of $S$ and so is $L^{*}=L \cap z^{-1} L$. By Lemma 1.2, $A=\{\sigma \in$ $\left.Y \mid L^{*} \cap S_{\sigma} \neq \varnothing\right\}$ is an $\cap$-large ideal of $Y$, and since $Y$ is nonsingular then $A$ is dense in $Y$. We now consider the following two cases:

Case 1. Suppose that $x \in S_{\alpha}$ and $y \in S_{\beta}$ with $\alpha \neq \beta$. Since $A$ is dense there exists $\delta \in A$ such that $\alpha \delta \neq \beta \delta$. Hence there exists a $t \in L^{*} \cap S_{\delta}$ such that $z * t \in L$ and $t \in L$. Since $\alpha \delta \neq \beta \delta$ then $S_{\alpha \dot{\delta}} \cap$ $S_{\beta \delta}=\varnothing$ and so $x * t \neq y * t$.

Case 2. Suppose that $x, y \in S_{\alpha}$ and define $[0, \alpha]=\{\sigma \in Y \mid 0 \leqq$ $\sigma \leqq \alpha\}$. Since $[0, \alpha]$ is a nonzero ideal of $Y$, then there exists $0 \neq \delta \in A \cap[0, \alpha]$. Thus there is a $t \in L^{*}$ with $t \in L$ and $z * t \in L$. Now $x * t \neq y * t$ for if otherwise then $\psi_{\alpha, \delta}(x) t=\psi_{\alpha, \delta}(y) t$. But $S_{\delta}$ is right cancellative so $\psi_{\alpha, \delta}(x)=\psi_{\alpha, \delta}(y)$. Since $\psi_{\alpha, \delta}$ is one-to-one then $x=y$ which is a contradiction.

Thus in both cases $L$ is a dense right ideal of $S$.

Corollary 2.3. Let $S$ be a sturdy semilattice $Y$ of right 
cancellative monoids $S_{\alpha}$. Then $S$ is right nonsingular if and only if $Y$ is nonsingular.

If each $\psi_{\alpha, \beta}(\alpha>\beta)$ is the trivial homomorphism; that is, it takes all elements to the identity, we say that $S$ has trivial multiplication.

THEOREM 2.4. Let $S$ be a strong semilattice $Y$ of monoids $S_{\alpha}$ and let $S$ have trivial multiplication. If $S$ is right nonsingular, then $\left|S_{\alpha}\right|>1$ implies $\alpha$ is an atom (a minimal nonzero element) of $Y$.

Proof. Let $\left|S_{\alpha}\right|>1$ and let $x, y \in S_{\alpha}$ with $x \neq y$. Also let $L$ be an $\cap$-large right ideal of $S$. Since $S$ is right nonsingular, $L$ is dense and so there exists $z \in S$ such that $x * z \neq y * z$ and $e_{\alpha} * z \in L$. We claim that if $z \in S_{\beta}$ then $\alpha \leqq \beta$. To see this we consider the following two cases:

Case 1. If $\alpha$ is not related to $\beta$ then $\alpha>\alpha \beta$ and $\beta>\alpha \beta$. Thus $x * z=\psi_{\alpha, \alpha \beta}(x) \psi_{\beta, \alpha \beta}(z)=e_{\alpha \beta} e_{\alpha \beta}=e_{\alpha \beta}$ and $y * z=\psi_{\alpha, \alpha \beta}(y) \psi_{\beta, \alpha \beta}(z)=$ $e_{\alpha \beta} e_{\alpha \beta}=e_{\alpha \beta}$. This is a contradiction since $x * z \neq y * z$.

Case 2. If $\beta \leqq \alpha$ then $x^{*} z=\psi_{\alpha, \beta}(x) \psi_{\beta, \beta}(z)=e_{\beta} z=z$ and $y * z=$ $\psi_{\alpha, \beta}(y) \psi_{\beta, \beta}(z)=e_{\beta} z=z$. Again this is a contradiction.

Let $B$ be an $\cap$-large ideal, $L^{*}$ and $z$ as before. Then $\alpha \leqq \beta$ implies $\alpha \beta=\alpha \in \beta$.

Finally, we suppose that $\alpha$ is not an atom of $Y$. Then there exists $\delta \in Y$ such that $0<\delta<\alpha$. Define $I=\{\sigma \in Y \mid \sigma \delta=0$ or $\sigma \leqq \delta\}$. It is easy to see that $I$ is an $\cap$-large ideal of $Y$ but $\alpha \notin I$ which is a contradiction.

THEOREM 2.5. Let $S$ be a strong semilattice $Y$ of right cancellative monoids $S_{\alpha}$. If $Y$ is nonsingular and $\left|S_{\alpha}\right|>1$ implies $\alpha$ is an atom of $Y$, then $S$ is right nonsingular.

Proof. Let $x \neq y, z \in S$ and let $L$ be an $\cap$-large right ideal of S. If $x \in S_{\alpha}$ and $y \in S_{\beta}$ with $\alpha \neq \beta$ by the same argument as in Theorem 2.2, Case 1 there exists $t \in L$ such that $x * t \neq y * t$ and $z * t \in L$. Hence assume that $x, y \in S_{\alpha}$, then since $\left|S_{\alpha}\right|>1, \alpha$ is an atom of $Y$ and $[0, \alpha]$ is a nonzero ideal of $Y$. Thus there exists $t \in L \cap S_{\alpha}$ such that $z * t \in L$ and $x * t \neq y * t$, for if otherwise $x=y$ since $S_{\alpha}$ is right cancellative and this would be a contradiction.

Note that if $\left|S_{\alpha}\right|>1$ implies $\alpha$ is an atom of $Y$, then $S$ has 
trivial multiplication.

CoROLlaRY 2.6. Let $S$ be a strong semilattice $Y$ of right cancellative monoids $S_{\alpha}$ and assume $S$ has trivial multiplication. Then $S$ is right nonsingular if and only if $E$ is nonsingular and $\left|S_{\alpha}\right|>1$ implies that $e_{\alpha}$ is an atom of $E$.

3. The maximal right quotient semigroup. Since McMorris [6] showed that the maximal right quotient semigroup of a semilattice of groups is a semilattice of groups, a natural question arises; which strong semilattices of semigroups have for their maximal right quotient semigroup a semilattice of groups? In this section, we let $S$ be a strong semilattice $Y$ of right cancellative principal right ideal monoids $S_{\alpha}$ with the linking homomorphisms onto.

LEMma 3.1. If $a S_{\alpha}$ is a dense principal right ideal of $S_{\alpha}$ then $\psi_{\alpha, \beta}(a) S_{\beta}$ is a dense principal right ideal of $S_{\beta}$ for $\alpha \geqq \beta$.

Proof. The proof is straightforward and is omitted.

Let $\alpha, \beta \in Y$ with $\alpha \geqq \beta$ and let $Q\left(S_{\alpha}\right), Q\left(S_{\beta}\right)$ be the maximal right quotient semigroup of $S_{\alpha}$ and $S_{\beta}$ respectively. The members of these equivalence classes will be denoted $[f]_{\alpha}$ and $[g]_{\beta}$ with the subscripts being dropped if there is no confusion.

We can extend $\psi_{\alpha, \beta}: S_{\alpha} \rightarrow S_{\beta}$ to a mapping $\phi_{\alpha, \beta}: Q\left(S_{\alpha}\right) \rightarrow Q\left(S_{\beta}\right)$ defined by $[f]_{\alpha} \rightarrow[\hat{f}]_{\beta}$ where if $f: a S_{\alpha} \rightarrow S_{\alpha}$ then $\hat{f}: \psi_{\alpha, \beta}(\alpha) S_{\beta} \rightarrow S_{\beta}$ is defined by $\psi_{\alpha, \beta}(\alpha) s \rightarrow \psi_{\alpha, \beta}(f(\alpha)) s$ for $s \in S_{\beta}$. Note that $\hat{f}$ is an $S_{\beta^{-}}$ homomorphism since if $t \in S_{\beta}$ then $\hat{f}\left(\psi_{\alpha, \beta}(\alpha) s\right) t=\left(\psi_{\alpha, \beta}(f(a)) s\right) t=$ $\psi_{\alpha, \beta}(f(a))(s t)=\hat{f}\left(\psi_{\alpha, \beta}(a)(s t)\right)=\hat{f}\left(\left(\psi_{\alpha, \beta}(a) s\right) t\right)$.

We next show that $\phi_{\alpha, \beta}$ is independent of the representative we choose from $[f]$. Hence let $[f]=[g]$, then $f$ and $g$ agree on a dense right ideal of $S_{\alpha}$, call it $D$, found in the intersection of their domains $D_{f}$ and $D_{g}$ respectively. Since $S_{\alpha}$ is a principal right ideal semigroup then $D_{f}=a S_{\alpha}, D_{g}=c S_{\alpha}$ and $D=x S_{\alpha}$ for some $a, c, x \in$ $S_{\alpha}$. Now $\phi_{\alpha, \beta}([f])=[\hat{f}]$ where $\hat{f}: \psi_{\alpha, \beta}(\alpha) S_{\beta} \rightarrow S_{\beta}$ defined by $\psi_{\alpha, \beta}(a) s \rightarrow$ $\psi_{\alpha, \beta}(f(a)) s$, and $\phi_{\alpha, \beta}([g])=[\hat{g}]$ where $\hat{g}: \psi_{\alpha, \beta}(c) S_{\beta} \rightarrow S_{\beta}$ defined by $\psi_{\alpha, \beta}(c) s \rightarrow \psi_{\alpha, \beta}(g(c)) s$. We claim $\hat{f}$ and $\hat{g}$ agree on the dense right ideal $\psi_{\alpha, \beta}(x) S_{\beta} \subseteq \psi_{\alpha, \beta}(\alpha) S_{\beta} \cap \psi_{\alpha, \beta}(c) S_{\beta}$. Since $x S_{\alpha} \subseteq \alpha S_{\alpha} \cap c S_{\alpha}$ it is easy to see that $\psi_{\alpha, \beta}(x) S_{\alpha} \subseteq \psi_{\alpha, \beta}(a) S_{\beta} \cap \psi_{\alpha, \beta}(c) S_{\beta}$. Furthermore, since $x S_{\alpha}$ is dense in $S_{\alpha}$ then by Lemma 3.1, $\psi_{\alpha, \beta}(x) S_{\beta}$ is dense in $S_{\beta}$. Hence let $\psi_{\alpha, \beta}(x) s \in \psi_{\alpha, \beta}(x) S_{\beta}$ then $\hat{f}\left(\psi_{\alpha, \beta}(x) s\right)=\widehat{f}\left(\psi_{\alpha, \beta}(x) \psi_{\alpha, \beta}(t)\right)$ where $t \in S_{\alpha}$ since $\psi_{\alpha, \beta}$ is onto. Since $\psi_{\alpha, \beta}$ is a semigroup homomorphism, it follows that $\hat{f}\left(\psi_{\alpha, \beta}(x) s\right)=\hat{f}\left(\psi_{\alpha, \beta}(x t)\right)=\psi_{\alpha, \beta}(f(x t))=\psi_{\alpha, \beta}(g(x t))=$ $\hat{g}\left(\psi_{\alpha, \beta}(x t)\right)=\hat{g}\left(\psi_{\alpha, \beta}(x) \psi_{\alpha, \beta}(t)\right)=\hat{g}\left(\psi_{\alpha, \beta}(x) s\right)$. Thus the claim is estab- 
lished.

TheOREM 3.2. Let $S=\bigcup_{\alpha \in Y} S$ be a strong semilattice $Y$ of right cancellative principal right ideal monoids $S_{\alpha}$ with $\psi_{\alpha, \beta}$ onto for $\alpha \geqq \beta \in Y$. If $T=\bigcup_{\alpha \in Y} Q\left(S_{\alpha}\right)$ with multiplication defined by

$$
[f]_{\alpha}[g]_{\beta}=\dot{\phi}_{\alpha, \alpha \beta}\left([f]_{\alpha}\right) \dot{\phi}_{\beta, \alpha \beta}\left([g]_{\beta}\right)
$$

where $[f]_{\alpha} \in Q\left(S_{\alpha}\right),[g]_{\beta} \in Q\left(S_{\beta}\right)$ and $\phi_{\alpha, \alpha \beta}, \phi_{\beta, \alpha \beta}$ are defined as above, then $T$ is a strong semilattice $Y$ of monoids $Q\left(S_{\alpha}\right)$.

Proof. Note that since $S_{\alpha} \cap S_{\beta}=\varnothing$ for $\alpha \neq \beta$ then $Q\left(S_{\alpha}\right) \cap$ $Q\left(S_{\beta}\right)=\varnothing$, and that $\phi_{\alpha, \alpha}$ is the identity mapping. We now show that $\phi_{\alpha, \beta}: Q\left(S_{\alpha}\right) \rightarrow Q\left(S_{\beta}\right)$ is a semigroup homomorphism. Let $[f],[g] \in$ $Q\left(S_{\alpha}\right)$ then we must show that $\phi_{\alpha, \beta}([f][g])=\phi_{\alpha, \beta}([f]) \phi_{\alpha, \beta}([g])$. To this end we let $\phi_{\alpha, \beta}([f])=[\hat{f}]$ and $\phi_{\alpha, \beta}([g])=[\hat{g}]$ where if $f: a S_{\alpha} \rightarrow S_{\alpha}$ and $g: c S_{\alpha} \rightarrow S_{\alpha}$ then $\hat{f}: \psi_{\alpha, \beta}(\alpha) S_{\beta} \rightarrow S_{\beta}$ defined by $\psi_{\alpha, \beta}(\alpha) s \rightarrow \psi_{\alpha, \beta}(f(\alpha)) s$ and $\hat{g}: \psi_{\alpha, \beta}(c) S_{\beta} \rightarrow S_{\beta}$ defined by $\psi_{\alpha, \beta}(c) s \rightarrow \psi_{\alpha, \beta}(g(c)) s$. Since $[f][g]=$ $[f g]$ where $f g: g^{-1}\left(\alpha S_{\alpha}\right) \rightarrow S_{\alpha}$ and $g^{-1}\left(\alpha S_{\alpha}\right)=\left\{x \in c S_{\alpha} \mid g(x) \in a S_{\alpha}\right\}$, then for some $h \in S_{\alpha}, h S_{\alpha}=g^{-1}\left(a S_{\alpha}\right)$ and so $\widehat{f g}: \psi_{\alpha, \beta}(h) S_{\beta} \rightarrow S_{\beta}$ defined by $\psi_{\alpha, \beta}(h) s \rightarrow \psi_{\alpha, \beta}(f g(h)) s$. Thus $\phi_{\alpha \beta}([f][g])=\phi_{\alpha, \beta}([\widehat{f g}])=[f g]$. On the other hand, $\phi_{\alpha, \beta}([f]) \dot{\phi}_{\alpha, \beta}([g])=[\hat{f}][\hat{g}]=[\hat{f} \hat{g}]$ where $\hat{f} \hat{g}: \hat{g}^{-1}\left(\psi_{\alpha, \beta}(a) S_{\beta}\right) \rightarrow$ $S_{\beta}$ and $\hat{g}^{-1}\left(\psi_{\alpha, \beta}(a) S_{\beta}\right)=\left\{y \in \psi_{\alpha, \beta}(c) S_{\beta} \mid \hat{g}(y) \in \psi_{\alpha, \beta}(a) S_{\beta}\right\}$. Hence we must show that $[\hat{f g}]=[\hat{f} \hat{g}]$; that is, $\widehat{f g}$ and $\hat{f} \hat{g}$ agree on a dense right ideal found in the intersection of their domains. Now $\psi_{\alpha, \beta}(h) S_{\beta} \subseteq$ $g^{-1}\left(\psi_{\alpha, \beta}(\alpha) S_{\beta}\right)$ for if $\psi_{\alpha, \beta}(h) s \in \psi_{\alpha, \beta}(h) S_{\beta}$ then $\psi_{\alpha, \beta}(h) s=\psi_{\alpha, \beta}(h) \psi_{\alpha, \beta}(t)$ where $t \in S_{\alpha}$ since $\psi_{\alpha, \beta}$ is onto. Thus $\psi_{\alpha, \beta}(h) s=\psi_{\alpha, \beta}(h t)=\psi_{\alpha, \beta}(c r)$ since $h t \in c S_{\alpha}$ and so $h t=c r$ for some $r \in S_{\alpha}$. Hence $\psi_{\alpha, \beta}$ being a semigroup homomorphism implies $\psi_{\alpha, \beta}(h) s=\psi_{\alpha, \beta}(c) \psi_{\alpha, \beta}(r) \in \psi_{\alpha, \beta}(c) S_{\beta}$. Now $\hat{g}\left(\psi_{\alpha, \beta}(h) s\right)=\psi_{\alpha, \beta}(g(h)) s=\psi_{\alpha, \beta}(g(h)) \psi_{\alpha, \beta}(t)=\psi_{\alpha, \beta}(g(h) t)=\psi_{\alpha, \beta}(g(h t))=$ $\psi_{\alpha, \beta}(a x)$ since $g(h t) \in a S_{\alpha}$ and so $g(h t)=a x$ for some $x \in S_{\alpha}$. Again since $\psi_{\alpha, \beta}$ is a semigroup homomorphism we have that $\hat{g}\left(\psi_{\alpha, \beta}(h) s\right)=$ $\psi_{\alpha, \beta}(a) \psi_{\alpha, \beta}(x) \in \psi_{\alpha, \beta}(a) S_{\beta}$. We now claim that $\widehat{f g}$ and $\hat{f} \hat{g}$ agree on $\psi_{\alpha, \beta}(h) S_{\beta}$. Let $\psi_{\alpha, \beta}(h) s \in \psi_{\alpha, \beta}(h) S_{\beta}$ then $\widehat{f g}\left(\psi_{\alpha, \beta}(h) s\right)=\psi_{\alpha, \beta}(f g(h)) s=$ $\psi_{\alpha, \beta}(f(g(h))) s=\hat{f}\left(\psi_{\alpha, \beta}(g(h))\right) s=\hat{f}\left(\psi_{\alpha, \beta}(g(h)) s\right)=\hat{f}\left(\hat{g}\left(\psi_{\alpha, \beta}(h)\right) s\right)=\hat{f} \hat{g}\left(\psi_{\alpha, \beta}(h) s\right)$.

Finally, we show that if $\alpha>\beta>\delta$ then $\phi_{\beta, \delta} \phi_{\alpha, \beta}=\phi_{\alpha, \delta}$. Let $[f] \in Q\left(S_{\alpha}\right)$ with $f: a S_{\alpha} \rightarrow S_{\alpha}$ and let $\phi_{\alpha, \delta}([f])=[\bar{f}] \in Q\left(S_{\delta}\right)$ where $\bar{f}: \psi_{\alpha, \delta}(a) S_{\delta} \rightarrow S_{\delta}$ defined by $\psi_{\alpha, \delta}(\alpha) s \rightarrow \psi_{\alpha, \beta}(f(a)) s$. Let $\phi_{\alpha, \beta}([f])=[\hat{f}] \epsilon$ $Q\left(S_{\beta}\right)$ where $\hat{f}: \psi_{\alpha, \beta}(a) S_{\beta} \rightarrow S_{\beta}$ defined by $\psi_{\alpha, \beta}(\alpha) t \rightarrow \psi_{\alpha, \beta}(f(a)) t$. Hence $\phi_{\beta, \delta}\left(\phi_{\alpha, \beta}([f])\right)=\phi_{\beta, \delta}([\hat{f}])=[\tilde{f}]$ where $\tilde{f}: \psi_{\beta, \delta}\left(\psi_{\alpha, \beta}(a)\right) S_{\delta} \rightarrow S_{\delta}$ is defined by $\psi_{\beta, \delta}\left(\psi_{\alpha, \beta}(a)\right) s \rightarrow \psi_{\beta, \delta}\left(\hat{f}\left(\psi_{\alpha, \beta}(a)\right)\right) s$. To see that $\tilde{f}=\bar{f}$, we note that $\psi_{\beta, \delta} \psi_{\alpha, \beta}=\psi_{\alpha, \delta}$ so $\psi_{\alpha, \delta}(a) S_{\delta}=\psi_{\beta, \delta}\left(\psi_{\alpha, \beta}(a)\right) S_{\delta}$. Hence if $\psi_{\beta, \delta}\left(\psi_{\alpha, \beta}(a)\right) s \epsilon$ $\psi_{\beta, \delta}\left(\psi_{\alpha, \beta}(a)\right) S_{\delta}$ then $\widetilde{f}\left(\psi_{\beta, \delta}\left(\psi_{\alpha, \beta}(a)\right) s\right)=\psi_{\beta, \delta}\left(\hat{f}\left(\psi_{\alpha, \beta}(a)\right)\right) s=\psi_{\beta, \delta}\left(\psi_{\alpha, \beta}(f(a))\right) s=$ 
$\psi_{\beta, \delta} \psi_{\alpha, \beta}(f(a)) s=\psi_{\alpha, \delta}(f(a)) s$.

THEOREM 3.3. Under the hypothesis of Theorem 3.2, $S$ can be embedded into $T$.

Proof. Define $\Phi: S \rightarrow T$ by $s \rightarrow\left[\lambda_{s}\right]$ where if $s \in S_{\alpha}$ then $\left[\lambda_{s}\right]_{\alpha} \in$ $Q\left(S_{\alpha}\right)$ and $\lambda_{s}: S_{\alpha} \rightarrow S_{\alpha}$ is defined by $t \rightarrow$ st. The mapping $\Phi$ is one-toone for suppose $\Phi(s)=\Phi(r)$ where $s \in S_{\alpha}$ and $r \in S_{\beta}$.

Case 1. If $\alpha \neq \beta$ then $\Phi(s) \neq \Phi(r)$ since $Q\left(S_{\alpha}\right) \cap Q\left(S_{\beta}\right)=\varnothing$.

Case 2. If $\alpha=\beta$ then $\left[\lambda_{s}\right]_{\alpha}=\left[\lambda_{r}\right]_{\alpha}$ and so $\lambda_{s}$ and $\lambda_{r}$ agree on a dense right ideal of $S_{\alpha^{\prime}}$ say $D$. Hence for $d \in D, s d=\lambda_{s}(d)=$ $\lambda_{r}(d)=r d$ and since $S_{\alpha}$ is right cancellative then $s=r$.

Next we show that $\Phi$ is a semigroup homomorphism. Let $x \in S_{\alpha}$, $y \in S_{\beta}$ then $\Phi(x * y)=\left[\lambda_{x * y}\right]_{\alpha \beta}$ where $\lambda_{x * y}: S_{\alpha \beta} \rightarrow S_{\alpha \beta}$ defined by $s \rightarrow(x * y) s=$ $\psi_{\alpha, \alpha \beta}(x) \psi_{\beta, \alpha \beta}(y) s . \quad$ Now $\Phi(x) \Phi(y)=\left[y_{x}\right]_{\alpha}\left[\lambda_{y}\right]_{\beta}=\phi_{\alpha, \alpha \beta}\left(\left[\lambda_{x}\right]_{\alpha}\right) \phi_{\beta, \alpha \beta}\left(\left[\lambda_{y}\right]_{\beta}\right)=$ $[\hat{f}][\hat{g}]=[\hat{f} \hat{g}]$ where $[\hat{f}],[\hat{g}] \in Q\left(S_{\alpha \beta}\right)$ and $\hat{f}: S_{\alpha \beta} \rightarrow S_{\alpha \beta}$ defined by $s \rightarrow$ $\psi_{\alpha, \alpha \beta}(x) s$ and $\hat{g}: S_{\alpha \beta} \rightarrow S_{\alpha \beta}$ defined by $s \rightarrow \psi_{\beta, \beta \alpha}(y) s$. If $s \in S_{\alpha \beta}$ then $\widehat{f} \hat{g}(s)=\hat{f}(\hat{g}(s))=\hat{f}\left(\psi_{\beta, \alpha \beta}(y) s\right)=\hat{f}\left(\psi_{\beta, \alpha \beta}(y)\right) s=\psi_{\alpha, \alpha \beta}(x) \psi_{\beta, \alpha \beta}(y) s=\lambda_{x * y}(s)$.

We identify $S$ with its image in $T$ and note that if $S$ is right nonsingular we have the diagram

$$
\begin{aligned}
& T \longrightarrow T / \psi_{T} \\
& \text { UII UU } \\
& S=S / \psi_{s} .
\end{aligned}
$$

THEOREM 3.4. Let $R=T / \psi_{T}$. Under the hypothesis of Theorem 3.2 and if $S$ is right nonsingular then $\psi_{R}=i_{R}$.

Proof. Suppose that $t_{1}^{*} \psi_{R} t_{2}^{*}$. Let $t_{1} \in t_{1}^{*}$ and $t_{2} \in t_{2}^{*}$ then $\left(t_{1} d\right) \psi_{T}\left(t_{2} d\right)$ for all $d \in D$ a dense right ideal of $S$. Hence for each $d \in D$ there exists $X_{d}$ dense in $S$ such that $t_{1} d x=t_{2} d x$ for all $x \in X_{d}$. Let $W=\bigcup_{d \in D} d X_{d}$, then $t_{1} w=t_{2} w$ for all $w \in W$. If $W$ is dense in $S$ then $t_{1} \psi_{T} t_{2}$ and so $t_{1}^{*}=t_{2}^{*}$. To see that $W$ is dense in $S$, we let $s_{1} \neq s_{2}, s_{3} \in S$. Since $D$ is dense then there exists $d \in D$ such that $s_{1} d \neq s_{2} d$ and $s_{3} d \in D$. Since $X_{s_{3} d}$ is dense then there exists $x \in X_{s_{3} d}$ such that $\left(s_{1} d\right) x \neq\left(s_{2} d\right) x$ and $\left(s_{3} d\right) x \in\left(s_{3} d\right) X_{s_{3} d}$. But then $s_{1}(d x) \neq s_{2}(d x)$ and $s_{3}(d x) \in W$. Since $d x \in D$ and $X_{d x}$ is dense there exists $y \in X_{d x}$ such that $s_{1}((d x) y) \neq s_{2}((d x) y)$ and $s_{3}((d x) y) \in X_{d x}$. But $W$ is a right ideal so $s_{3}((d x) y) \in W$ with $(d x) y \in W$. This shows that $W$ is dense in $S$. 
A right Ore semigroup is a right cancellative semigroup all of whose nonzero right ideals are $\cap$-large. The maximal right quotient semigroup of a right Ore semigroup $R$ is a group $Q(R)=$ $\left\{a b^{-1} \mid a, b \in R\right\}[2]$.

THEOREM 3.5. Let $S=\bigcup_{\alpha \in Y} S_{\alpha}$ be a strong semilattice $Y$ of principal right ideal Ore monoids $S_{\alpha}$ with $\psi_{\alpha, \beta}$ onto for $\alpha \geqq \beta \in Y$. If $S$ is right nonsingular then $Q(S)$ is a semilattice of groups.

Proof. By Theorem 3.2, $T=\bigcup_{\alpha \in Y} Q\left(S_{\alpha}\right)$ is a strong semilattice and since each $Q\left(S_{\alpha}\right)$ is a group then $T$ is a semilattice $Y$ of groups $Q\left(S_{\alpha}\right)$ and so regular with idempotents in the center of $T[1, \mathrm{pp}$. 128-129]. Hence $T / \psi_{T}$ is regular and its idempotents are in the center of $T / \psi_{T}$, which makes $T / \psi_{T}$ a semilattice of groups. McMorris [6] showed that $Q\left(T / \psi_{T}\right)$ is also a semilattice of groups. By Theorem 3.4, $Q(S) \approx Q\left(T / \psi_{T}\right)$ and so is a semilattice of groups.

THEOREM 3.6. Under the hypothesis of Theorem 3.5, T/ $\psi_{T}$ can be taken to be the union of the same semilattice $Y$ of groups.

Proof. Since $T=\bigcup_{\alpha \in Y} Q\left(S_{\alpha}\right)$ where each $Q\left(S_{\alpha}\right)$ is a group, we let $e_{\alpha}=\left[e_{\alpha}\right] \in Q\left(S_{\alpha}\right)$. If $e_{\alpha} \psi_{T} e_{\beta}$ when $\alpha \neq \beta$ then $e_{\alpha} * x=e_{\beta} * x$ for all $x \in L$ an $\cap$-large right ideal of $S$. Since $S$ is right nonsingular then $Y$ is right nonsingular by Theorem 2.1. Furthermore, $A=\{\sigma \in Y \mid L \cap$ $S_{o} \neq \neq \varnothing$ is dense in $Y$. Hence since $\alpha \neq \beta$ there exists $\delta \in A$ such that $\alpha \delta \neq \beta \delta$. Let $t \in L \cap S_{\delta}$ then $e_{\alpha} * t=e_{\beta} * t$ which implies that $e_{\alpha \hat{\delta}} \psi_{\hat{\delta}, \alpha \hat{\delta}}(t)=e_{\beta \hat{\delta}} \psi_{\tilde{\delta}, \beta \delta}(t)$ or that $\phi_{\delta, \alpha \delta}(t)=\phi_{\hat{\delta}, \beta \hat{\delta}}(t)$. This is a contradiction since for $\alpha \delta \neq \beta \delta, Q\left(S_{\alpha \bar{\delta}}\right) \cap Q\left(S_{\beta \delta}\right) \neq \varnothing$. Hence $e_{\alpha} \psi_{T} \neq e_{\beta} \psi_{T}$ when $\alpha \neq \beta$. Thus in $T / \psi_{T}$ there are at least as many idempotents as there are in $T$. Now suppose that $g \psi_{T}$ is an idempotent of $T / \psi_{T}$. Since $g \in Q\left(S_{\alpha}\right)$ a group then $g \psi_{T} \in Q\left(S_{\alpha}\right) / \psi_{T}$, also a group. The only idempotent of $Q\left(S_{\alpha}\right) / \psi_{T}$ is $e_{\alpha} \psi_{T}$ so $g \psi_{T}=e_{\alpha} \psi_{T}$. Hence in $T / \psi_{T}$ there are no new idempotents.

Hinkle [2] showed that $Q\left(T / \psi_{T}\right)$ is a semilattice $Q(Y)$ of groups. Thus $Q(S)$ is a semilattice $Q(Y)$ of groups where $Y$ is the semilattice of both $S$ and $T / \psi_{T}$. The next theorem is a restatement of the above results.

THEOREM 3.7. Let $S$ be a strong semilattice $Y$ of principal right ideal Ore monoids with onto linking homomorphisms. If $S$ is right nonsingular then $Q(S)$ is a semilattice $Q(Y)$ of groups. 


\section{REFERENCES}

1. A. H. Clifford and G. B. Preston, The algebraic theory of semigroups, Vol. 1, Math. Surveys of the Amer. Math. Soc., (Providence, R. I., 1961).

2. C. V. Hinkle, Jr., Semigroups of right quotients of a semigroup which is a semilattice of groups, Semigroup Forum., 5 (1972), 167-173.

3. C. S. Johnson, Jr. and F. R. McMorris, Nonsingular semilattices and semigroups, Czechoslovak Math. J., 26 (101) 2 (1976), 280-282.

4. — Commutative nonsingular semigroups submitted.

5. A. M. Lopez, Jr. and J. K. Luedeman, The bicommutator of the injective hull of a nonsingular semigroup, Semigroup Forum, 12 (1976), 71-77.

6. F. R. McMorris, The quotient semigroup of a semigroup that is a semilattice of groups, Glasgow Math. J., 12 (1971), 18-23.

7. —, On quotient semigroups, J. Math. Sci., 7(1972), 48-56.

8. - The singular congruence and the maximal quotient semigroup, Canad. Math. Bull., 15 (1972), 301-303.

9. M. Petrich, Introduction to semigroups, Charles E. Merrill Publishing Co., (Columbus, Ohio, 1973).

Received November 29, 1976 and in revised form March 9, 1977. This paper contains part of a doctoral dissertation written under the direction of Professor John $\mathrm{K}$. Luedeman at Clemson University.

LOYOLA UNIVERSITY

New ORleans, LA 70118 



\section{PACIFIC JOURNAL OF MATHEMATICS}

\section{EDITORS}

RICHARD ARENS (Managing Editor)

University of California

Los Angeles, CA 90024

Charles W. Curtis

University of Oregon

Eugene, OR 97403

C. C. MOORE

University of California

Berkeley, CA 94720

\section{J. DugundJI}

Department of Mathematics

University of Southern California

Los Angeles, CA 90007

R. FinN and J. Milgram

Stanford University

Stanford, CA 94305

ASSOCIATE EDITORS
E. F. BECKENBACH
B. H. NeumanN
F. WOLF
K. YOSHIDA

\section{SUPPORTING INSTITUTIONS}

UNIVERSITY OF BRITISH COLUMBIA CALIFORNIA INSTITUTE OF TECHNOLOGY

UNIVERSITY OF CALIFORNIA

MONTANA STATE UNIVERSITY

UNIVERSITY OF NEVADA, RENO

NEW MEXICO STATE UNIVERSITY

OREGON STATE UNIVERSITY

UNIVERSITY OF OREGON

OSAKA UNIVERSITY

\author{
UNIVERSITY OF SOUTHERN CALIFORNIA \\ STANFORD UNIVERSITY \\ UNIVERSITY OF HAWAII \\ UNIVERSITY OF TOKYO \\ UNIVERSITY OF UTAH \\ WASHINGTON STATE UNIVERSITY \\ UNIVERSITY OF WASHINGTON \\ AMERICAN MATHEMATICAL SOCIETY
}

The Supporting Institutions listed above contribute to the cost of publication of this Journal, but they are not owners or publishers and have no responsibility for its content or policies.

Mathematical papers intended for publication in the Pacific Jaurnal of Mathematics should be in typed form or offset-reproduced, (not dittoed), double spaced with large margins. Please do not use built up fractions in the text of your manuscript. You may however, use them in the displayed equations. Underline Greek letters in red, German in green, and script in blue. The first paragraph or two must be capable of being used separately as a synopsis of the entire paper. Items of the bibliography should not be cited there unless absolutely necessary, in which case they must be identified by author and Journal, rather than by item number. Manuscripts, in triplicate, may be sent to any one of the editors. Please classify according to the scheme of Math. Reviews, Index to Vol. 39. All other communications should be addressed to the managing editor, or Elaine Barth, University of California, Los Angeles, California, 90024.

The Pacific Journal of Mathematics expects the author's institution to pay page charges, and reserves the right to delay publication for nonpayment of charges in case of financial emergency.

100 reprints are provided free for each article, only if page charges have been substantially paid. Additional copies may be obtained at cost in multiples of 50 .

The Pacific Journal of Mathematics is issued monthly as of January 1966. Regular subscription rate: $\$ 7200$ a year (6 Vols., 12 issues). Special rate: $\$ 36.00$ a year to individual members of supporting institutions.

Subscriptions, orders for back numbers, and changes of address should be sent to Pacific Journal of Mathematics, 103 Highland Boulevard, Berkeley, California, 94708.

PUBLISHED BY PACIFIC JOURNAL OF MATHEMATICS, A NON-PROFIT CORPORATION

Printed at Kokusai Bunken Insatsusha (International Academic Printing Co., Ltd.). 8-8, 3-chome, Takadanobaba, Shinjuku-ku, Tokyo 160, Japan.

Copyright (C) 1975 by Pacific Journal of Mathematics Manufactured and first issued in Japan 


\section{Pacific Journal of Mathematics}

\section{Vol. 71, No. $2 \quad$ December, 1977}

Krishnaswami Alladi and Paul Erdős, On an additive arithmetic

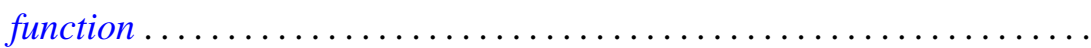

James Bailey and Dale Rolfsen, An unexpected surgery construction of a

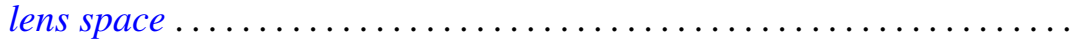

Lawrence James Brenton, On the Riemann-Roch equation for singular

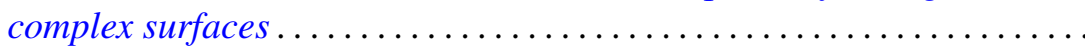

James Glenn Brookshear, Projective ideals in rings of continuous

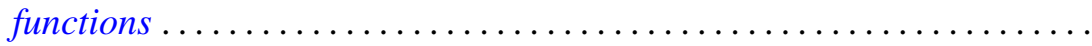

Lawrence Gerald Brown, Stable isomorphism of hereditary subalgebras of

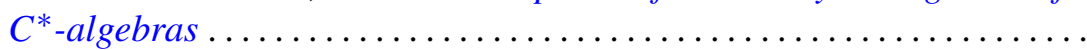

Lawrence Gerald Brown, Philip Palmer Green and Marc Aristide Rieffel, Stable isomorphism and strong Morita equivalence of $C^{*}$-algebras....

N. Burgoyne, Robert L. Griess, Jr. and Richard Lyons, Maximal subgroups

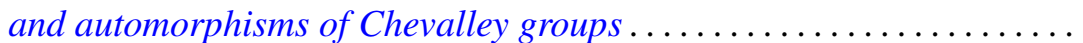
365

Yuen-Kwok Chan, Constructive foundations of potential theory .... 405

Peter Fletcher and William Lindgren, On $w \Delta$-spaces, $w \sigma$-spaces and

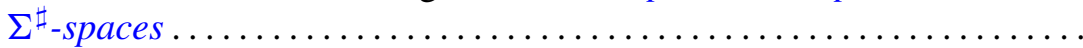

Louis M. Friedler and Dix Hayes Pettey, Inverse limits and mappings of

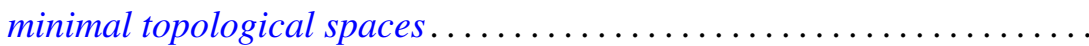

Robert E. Hartwig and Jiang Luh, A note on the group structure of unit regular ring elements.

I. Martin (Irving) Isaacs, Real representations of groups with a single involution ...

Nicolas P. Jewell, The existence of discontinuous module derivations . .

Antonio M. Lopez, The maximal right quotient semigroup of a strong semilattice of semigroups .......................

Dennis McGavran, $T^{n}$-actions on simply connected $(n+2)$-manifolds

Charles Anthony Micchelli and Allan Pinkus, Total positivity and the exact $n$-width of certain sets in $L^{1}$.

Barada K. Ray and Billy E. Rhoades, Fixed point-theorems for mappings with a contractive iterate .......................

Fred Richman and Elbert A. Walker, Ext in pre-Abelian categories. .

Raymond Craig Roan, Weak* generators of $H^{\infty}$ and $l^{1}$..

Saburou Saitoh, The exact Bergman kernel and the kernels of Szegö type...

Kung-Wei Yang, Operators invertible modulo the weakly compact 